\title{
PAPER
}

\section{Entanglement entropy and out-of-time-order correlator in the long-range Aubry-André-Harper model}

To cite this article: Nilanjan Roy and Auditya Sharma 2021 J. Phys.: Condens. Matter 33334001

View the article online for updates and enhancements.

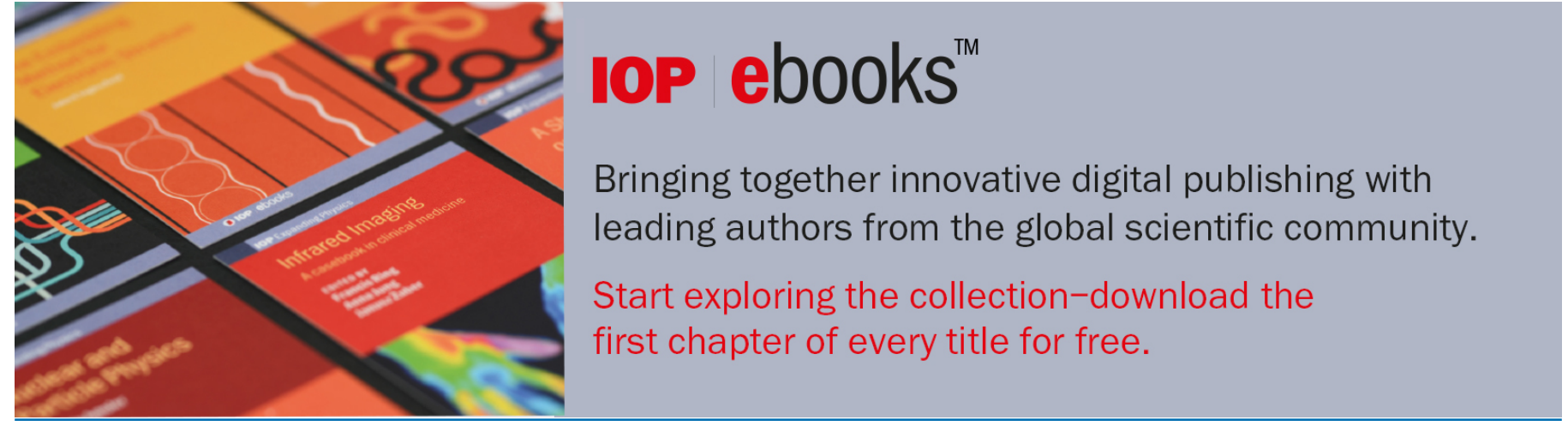

This content was downloaded from IP address 14.139 .128 .34 on 28/09/2021 at 09:23 


\title{
Entanglement entropy and out-of-time-order correlator in the long-range Aubry-André-Harper model
}

\author{
Nilanjan Roy ${ }^{1,2}$ and Auditya Sharma ${ }^{1, *}$ (1) \\ 1 Department of Physics, Indian Institute of Science Education and Research, Bhopal, Madhya Pradesh \\ 462066, India \\ ${ }^{2}$ Department of Physics, Indian Institute of Science, Bangalore 560012, India \\ E-mail: auditya@iiserb.ac.in
}

Received 8 February 2021, revised 17 May 2021

Accepted for publication 1 June 2021

Published 25 June 2021

\begin{abstract}
We investigate the nonequilibrium dynamics of entanglement entropy and out-of-time-order correlator (OTOC) of noninteracting fermions at half-filling starting from a product state to distinguish the delocalized, multifractal (in the limit of nearest neighbor hopping), localized and mixed phases hosted by the quasiperiodic Aubry-André-Harper (AAH) model in the presence of long-range hopping. For sufficiently long-range hopping strength a secondary logarithmic behavior in the entanglement entropy is found in the mixed phases whereas the primary behavior is a power-law the exponent of which is different in different phases. The saturation value of entanglement entropy in the delocalized, multifractal and mixed phases depends linearly on system size whereas in the localized phase (in the short-range regime) it is independent of system size. The early-time growth of OTOC shows very different power-law behaviors in the presence of nearest neighbor hopping and long-range hopping. The late time decay of OTOC leads to noticeably different power-law exponents in different phases. The spatial profile of OTOC and its system-size dependence also provide distinct features to distinguish phases. In the mixed phases the spatial profile of OTOC shows two different dependences on space for small and large distances respectively. Interestingly the spatial profile contains large fluctuations at the special locations related to the quasiperiodicity parameter in the presence of multifractal states.
\end{abstract}

Keywords: quantum dynamics, out-of-time-order correlator, entanglement entropy, long-range Aubry-André-Harper model

(Some figures may appear in colour only in the online journal)

\section{Introduction}

The nature of correlations between different parts of a system is of fundamental interest in physics. Entanglement entropy has been a popular measure of quantum correlations in manybody systems [1]. The study of entanglement in stationary, equilibrium and nonequilibrium states has proven to be insightful in a wide variety of contexts [2-6]. In recent years, out-of-time-order correlators or OTOC, which have emerged as a useful probe of quantum chaos [7], have gained

\footnotetext{
* Author to whom any correspondence should be addressed.
}

importance in a diverse set of fields ranging from high energy physics [8-11] to condensed matter physics [12-17] to quantum information $[18,19]$. Devised originally as a theoretical measure [20,21], considerable excitement has been generated from the recent experimental measurement of OTOC using nuclear magnetic resonance [22-24] and trapped ions [25, 26]. The OTOC is generically defined as:

$$
C(x, t)=\left\langle[\hat{W}(x, t), \hat{V}(0,0)]^{\dagger}[\hat{W}(x, t), \hat{V}(0,0)]\right\rangle,
$$

where $\hat{W}$ and $\hat{V}$ are arbitrary local operators separated by a displacement $x$ and commute at $t=0$. Here $\langle\cdot\rangle$ typically refers 
to a thermal average, although the expectation value in specific states may also be of interest. Choosing both $W$ and $V$ to be both Hermitian and unitary is particularly advantageous as equation (1) reduces to the compact expression:

$$
C(x, t)=2(1-\operatorname{Re}[F(x, t)]),
$$

where $F(x, t)=\langle\hat{W}(x, t) \hat{V}(0) \hat{W}(x, t) \hat{V}(0)\rangle$. At $t=0 C(x, t)$ is zero. Then it increases for $t>0$ due to non-commutativity of $\hat{W}(x, t)$ and $\hat{V}(0)$.

Models that exhibit localization are a natural setup for investigation of OTOC, in condensed matter systems. A particularly important class of such models is the family of models with quasi-periodic disorder, that have sustained interest over several decades [27-30]. Unlike with Anderson localization where even an infinitesimal random disorder results in localization, a quasiperiodic disorder of finite strength is essential for localization of a single particle even in one dimension $[31,32]$. There has been a revival of interest in quasiperiodic systems since their experimental realization using ultra-cold atoms [33-36]. Furthermore the possibility of many-body localization in such models has triggered a lot of interest both from a theoretical [37-39] and an experimental [40] perspective. Apart from the delocalized and localized phases, quasiperiodic systems can also host other nonergodic phases $[41,42]$ with their characteristic properties. In this study, we numerically probe the different phases using quantum dynamics of OTOC. We also study the quantum dynamics of entanglement entropy to complement and contrast against OTOC.

If $C(x, t)$ remains non-zero for an extended period of time one says that the system has 'scrambled'. For early time approach to scrambling one expects $C(x, t) \sim \mathrm{e}^{\lambda_{\text {quant }}\left(t-x / v_{\mathrm{B}}\right)}$ where $\lambda_{\text {quant }}$ is the 'quantum Lyapunov exponent' which is bounded by $\lambda_{\text {quant }} \leqslant 2 \pi k_{\mathrm{B}} T / \hbar$ as conjectured in [9]. $v_{\mathrm{B}}$ is called the 'Butterfly velocity' which is also bounded by the Lieb-Robinson bound [43]. Quantum systems in which $\lambda_{\text {quant }}$ approaches its bound are called fast scramblers [44, 45]. However, many condensed matter systems exhibit a much slower growth and hence are called slow scramblers. This includes the many-body localized systems showing a power law growth $[13,15,16,46]$ which itself may be contrasted with Anderson localized systems where $C(x, t)$ is expected to be a constant [16]. It should be noted that $\lambda_{\text {quant }}$, although inspired by classical chaos is quite different from its classical counterpart $\lambda_{\mathrm{L}}$ that characterizes chaotic motion in classical systems $[14,47,48]$. The OTOC corresponding to classical chaos was found to grow as $C(t)=\left\langle[q(t), p]^{2}\right\rangle \sim \mathrm{e}^{2 \lambda_{\mathrm{L}} t}$, where $\lambda_{\mathrm{L}}$ may become arbitrarily large.

Also the late time dynamics of $C(x, t)$ has turned out to be quite interesting. An inverse power-law behavior has been seen in integrable quantum spin chains $[49,50]$ and many-body localized systems [13]. Recently late time behavior of $C(x, t)$ has been proposed as a diagnostic to distinguish regular and chaotic quantum systems $[51,52]$. Although OTOC has been studied extensively in quantum systems, not many disordered integrable models have been addressed $[53,54]$ in the context of the delocalization-localization transition. In addition to studies that look at the evolution of an initial thermal state, studies involving an initial product state in a nonequilibrium setting have also been carried out $[15,53,55,56]$. Here we study OTOC starting from a CDW-type initial product state. We also study entanglement entropy which has been one of the most popular tools to characterize different many-body phases, especially in disordered quantum systems [5].

This paper is organized as follows. In section 2 we introduce the model and briefly discuss the various single particle phases shown by it $[41,42]$. In section 3 we describe the results obtained from the nonequilibrium dynamics of the entanglement entropy. In section 4 we study the nonequilibrium dynamics of OTOC. This section consists of two subsections: subsection 4.1 where we briefly describe the formalism for noninteracting fermions and subsection 4.2 where we discuss the results for our model. Finally we conclude in section 5.

\section{The model}

The one dimensional long-range Harper (LRH) model is given by the Hamiltonian:

$$
H=-\sum_{i<j}^{N}\left(\frac{J}{r_{i j}^{\sigma}} \hat{c}_{i}^{\dagger} \hat{c}_{j}+\text { H.c. }\right)+\lambda \sum_{i=1}^{N} \cos \left(2 \pi \alpha i+\theta_{\mathrm{p}}\right) \hat{n}_{i},
$$

where $\hat{c}_{i}^{\dagger}\left(\hat{c}_{i}\right)$ represents the single particle creation (destruction) operator at site $i$ and $\hat{n}_{i}=\hat{c}_{i}^{\dagger} \hat{c}_{i}$, the number operator acting at site $i$. We consider a lattice of total number of sites $N$, where $r_{i j}$ is the geometric distance between the sites $i$ and $j$ in an open chain. Here $\lambda$ is the strength of the quasi-periodic potential with the quasiperiodicity parameter $\alpha$ which is a Diophantine irrational number [57] e.g. $\alpha_{\mathrm{g}}=(\sqrt{5}-1) / 2$, $\alpha_{\mathrm{s}}=(\sqrt{2}-1), \alpha_{\mathrm{b}}=(\sqrt{13}-3) / 2$ etc $[58,59]$, also known as the 'golden mean', 'silver mean', 'bronze mean' etc. $\theta_{\mathrm{p}}$ is an arbitrary global phase. The strength of the long range hopping is controlled by $J$ and the long range parameter in the hopping $\sigma$. We set our units such that $J=1$ throughout this article. In the $\sigma \rightarrow \infty$ limit, this model is the wellknown Aubry-André-Harper (AAH) model [31, 32]. The AAH model has a self-dual point $\lambda=2 J$, where the model in position space maps to itself in momentum space. As a consequence, all the eigenstates are delocalized in position space for $\lambda<2 J$ and localized for $\lambda>2 J$ [57]. Some fillingfraction dependent properties of the AAH model have also been reported $[42,60]$.

The single particle phase diagram of the LRH model has been chalked out recently [41, 42]. Along with the delocalized and localized phases the phase diagram contains mixed phases where a certain fraction of delocalized eigenstates coexists with multifractal or localized eigenstates. For the 'golden mean' $\alpha_{\mathrm{g}}$ the mixed phases can be denoted as $P_{q}(q=$ $1,2,3, \ldots)$ where $\alpha_{\mathrm{g}}^{q}$ fraction of eigenstates are delocalized and $\left(1-\alpha_{\mathrm{g}}^{q}\right)$ fraction of eigenstates are multifractal or localized depending on whether $\sigma<1$ or $\sigma>1$. Hence $P_{q}$ phases for $\sigma<1$ contain the delocalized-multifractal (DM) edges. $P_{q}$ phases for $\sigma>1$ contain the delocalized-localized (DL) 


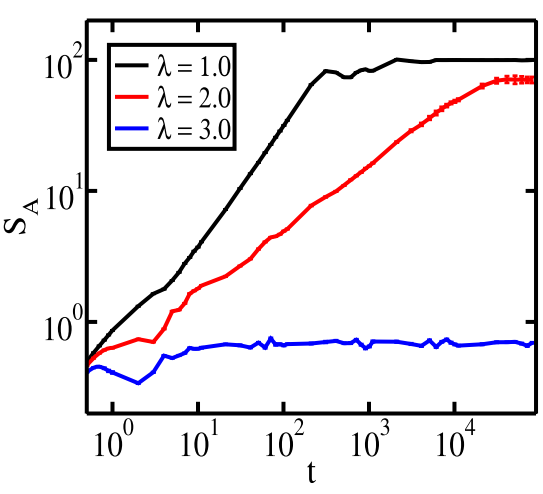

(a)

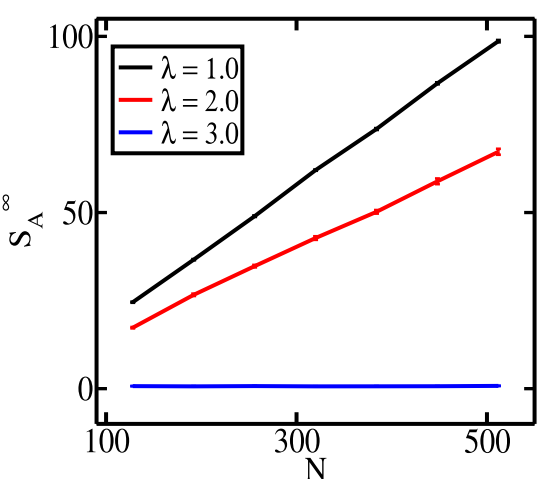

(b)

Figure 1. Entanglement entropy in the AAH model. (a) The dynamics of the half-chain entanglement entropy $S_{\mathrm{A}}$ with increasing values of $\lambda$ for free fermions at half-filling. Here system size $N=512$. (b) The system size $N$ dependence of the saturation value of the half-chain entanglement entropy $S_{\mathrm{A}}^{\infty}$ of free fermions at half-filling for increasing values of $\lambda$. For all the plots, total number of $\theta_{\mathrm{p}}$ realizations is 100 with quasi-periodicity fixed to be $\alpha_{\mathrm{g}}$.

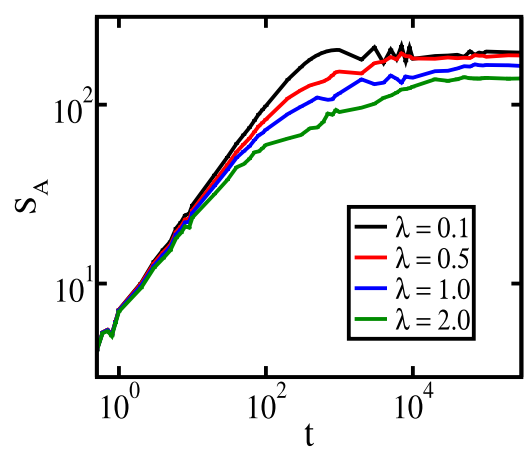

(a)

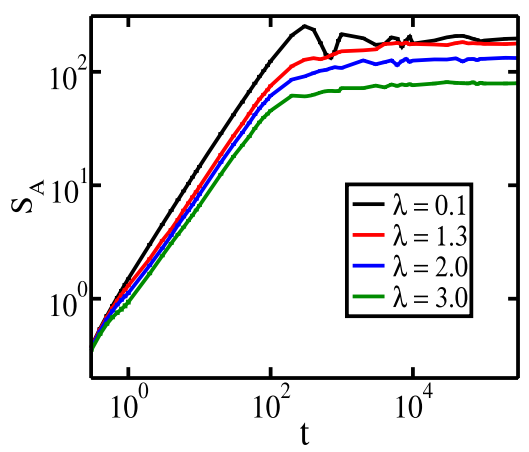

(b)

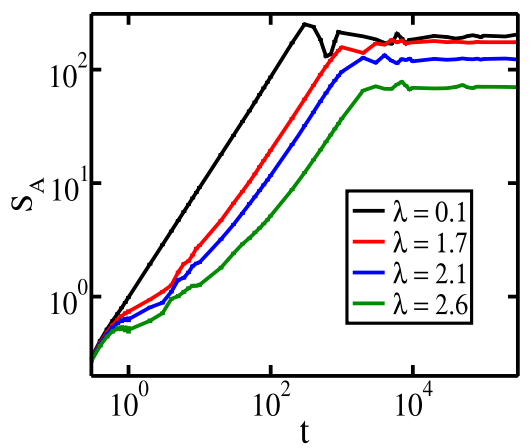

(c)

Figure 2. (a)-(c) The dynamics of the half-chain entanglement entropy $S_{\mathrm{A}}$ with increasing values of $\lambda$ for free fermions at half-filling and for $\sigma=0.5,1.5$ and 3.0 respectively. For all the plots system size $N=1024$.

edges, also known as mobility edges. For the present numerical study we have chosen some specific $(\lambda, \sigma)$ values. For $\sigma=0.5$, we consider $\lambda=0.1,0.5,1.0,2.0$ which correspond to the delocalized, $P_{1}, P_{2}$ and $P_{3}$ phases (with DM edge) respectively. For $\sigma=1.5$, we look at $\lambda=0.1,1.3,2.0,3.0$ which correspond to the delocalized, $P_{1}, P_{2}$ and $P_{3}$ phases (with DL edge) respectively. For $\sigma=3.0$, we look at $\lambda=0.1,1.7,2.1,2.5,5.0$ which correspond to the delocalized, $P_{1}, P_{2}, P_{3}$ phases (with DL edge) and localized phases respectively with $\sigma=3.0$ being essentially the short-range limit. Next we discuss the nonequilibrium dynamics of free fermions in the AAH and LRH models.

\section{Entanglement entropy}

The study of out-of-equilibrium properties of disordered quantum systems has been proved to be a very efficient tool to detect delocalized and localized phases. The system is initially prepared in a suitable state, and the properties of the time-evolved state are tracked. Since a charge density wave (CDW) type of state (for fermions at half-filling) is easily prepared in experiments involving ultra-cold atoms, we consider a CDW state as the initial state in our study. The initial state can be written as:

$$
\left|\Psi_{\text {in }}\right\rangle=\prod_{i=1}^{N / 2} \hat{c}_{2 i}^{\dagger}|0\rangle .
$$

We are mainly interested in the dynamics of entanglement entropy and the OTOC which are of current interest for integrable disordered quantum systems [61]. In this section we discuss the dynamics of entanglement entropy. OTOC will be discussed in the following section. We will stick to the quasiperiodicity parameter $\alpha_{\mathrm{g}}=(\sqrt{5}-1) / 2$ unless otherwise mentioned.

When the overall state of the system is pure, entanglement entropy is simply given by $S_{\mathrm{A}}=-\operatorname{Tr}\left(\rho_{\mathrm{A}} \ln \rho_{\mathrm{A}}\right)$ where $\rho_{\mathrm{A}}$ is the reduced density matrix of the subsystem $\mathrm{A}$. We calculate the dynamics of the half-chain entanglement entropy using free fermionic techniques $[62,63]$ that allow for the study of significantly large system sizes. In the AAH model, the growth of $S_{\mathrm{A}}$ is ballistic in time in the delocalized phase $(\lambda=1)$ and (almost) diffusive at the critical point $(\lambda=2)$ whereas there is essentially no growth in the localized phase $(\lambda=3)$ as shown in figure 1(a). These results are in agreement with those of an earlier study of quench dynamics in the AAH model [64]. Figure 1(b) shows that the saturation value $S_{\mathrm{A}}^{\infty}$ scales linearly with system sizes $\left(S_{\mathrm{A}}^{\infty} \propto N\right)$ at $\lambda=1$ and $\lambda=2$, while 


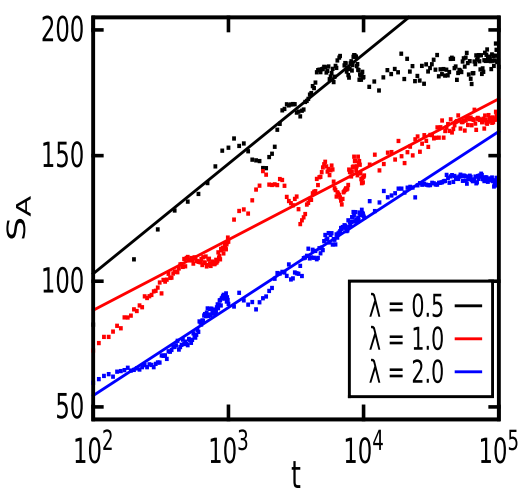

(a)

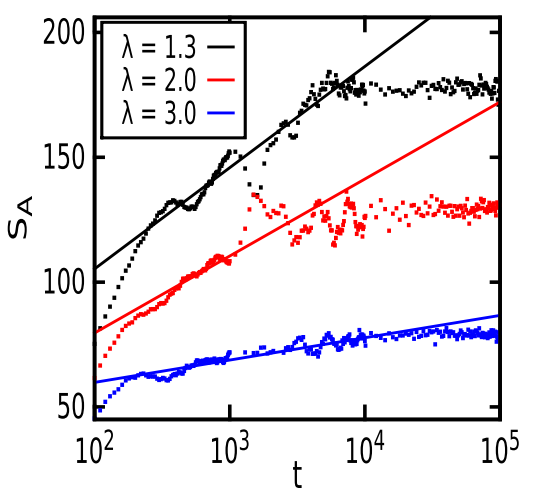

(b)

Figure 3. (a) The secondary logarithmic growth of the half-chain entanglement entropy $S_{\mathrm{A}}$ in the LRH model for $\sigma=0.5$ and $\lambda=0.5,1.0,2.0$ for which the best fits are $18.98 \ln t+15.64,12.18 \ln t+32.38$ and $15.21 \ln t-15.54$ respectively. (b) Similar plots for $\sigma=1.5$ and $\lambda=1.3,2.0,3.0$ for which best fits are $17.61 \ln t+24.15,13.39 \ln t+17.94$ and $3.89 \ln t+41.93$ respectively. The solid lines are best fits whereas the scattered points represent the corresponding data-points. The $x$-axis is in the log scale. For all the plots system size $N=1024$ and fermionic filling fraction is $1 / 2$.

$S_{\mathrm{A}}^{\infty} \propto N^{0}$ for $\lambda=3$. Also we have checked that these results remain independent of the choice of the quasiperiodicity parameter $\alpha$.

The plots of $S_{\mathrm{A}}$ as a function of time for the LRH model are shown in figures 2(a)-(c) for increasing values of $\lambda$ and $\sigma=0.5,1.5$ and 3.0 respectively. In the plots for $\sigma=0.5$ and $\sigma=1.5$ each, $S_{\mathrm{A}}$ shows two different behaviors with time which can be noticed both in figures 2(a) and (b). In figure 2(a) after the initial transient a power-law growth is found followed by a secondary logarithmic growth (see figure 3(a)). The secondary growth appears presumably due to the presence of the DM edge. It is to be noted that the secondary growth is absent for $\lambda=0.1$ for which all the eigenstates are delocalized. The primary growth in the dynamics of $S_{\mathrm{A}}$ can be fitted with a function $S_{\mathrm{A}}(t)=c_{1} t^{\beta}+c_{2}$ to extract the values of the power-law exponent $\beta$. For $\lambda=0.1, \beta$ turns out to be 0.53 . For other values of $\lambda=0.5,1.0,2.0$ which correspond to mixed phases with DM edges, $\beta=0.45,0.38$ and 0.31 respectively.

In figure 2(b) for $\sigma=1.5$ a primary power-law growth and a subsequent secondary logarithmic (see figure 3(b)) growth is observed. For $\sigma=1.5, \lambda=0.1$ corresponds to the delocalized phase whereas $\lambda=1.3,2.0,3.0$ here correspond to mixed phases with DL edges. For $\lambda=0.1,1.3,2.0$ and 3.0, the power-law exponent $\beta=0.89,0.82,0.80$ and 0.76 respectively. The secondary growth is again absent for $\lambda=0.1$ for which there is no DL edge. For $\sigma=3.0$ the secondary growth is absent as seen from figure 2(c) since the LRH model approaches the short-range AAH limit at this point. For $\lambda=0.1$ the growth of $S_{\mathrm{A}}$ happens ballistically as $\beta=1.0$ as in the delocalized phase of the short-range AAH model. For $\lambda=1.7,2.1,2.6$ the system is in the mixed phases with the DL edges. In the mixed phases the growth of $S_{\mathrm{A}}$ is initially less sensitive to the delocalized eigenstates due to the short-rangeness of the system. After some time the delocalized eigenstates start to dominate as indicated by the increasing change of rate of $S_{\mathrm{A}}$ in figure 2(c). Right before reaching saturation the power-law fit provides $\beta=0.84,0.82,0.79$ for $\lambda=1.7,2.1,2.6$ respectively. The secondary logarithmic growth for $\sigma=0.5,1.5$ are depicted in figures 3(a) and (b) respectively where the plots are fitted with the function $S_{\mathrm{A}}(t)=a_{1} \ln t+b_{1}$. Lots of intrinsic fluctuations can be seen in the plots due to the quasiperiodicity in the system. The secondary logarithmic growth tends to vanish in the short-range limit of hopping as these are barely seen for $\sigma=3.0$ (see figure 2(c)). Logarithmic growth of entanglement entropy has been seen recently in a few noninteracting randomly disordered systems $[61,65]$ and also longrange interacting systems [66]. The logarithmic behavior in the quasiperiodically disordered long-ranged LRH model is attributed to the presence of mixed phases in the longrange regime. This feature is not found in the short-range regime, and in general, in the absence of mixed phases.

We notice that the power-law exponent $\beta$ is larger for $\sigma>1$ as compared to $\sigma<1$. This counter-intuitive behavior of the power-law exponent in the entanglement growth has been addressed earlier in a clean free fermionic long-range model [67] and our work shows that this feature is robust against quasiperiodic disorder. It is noteworthy that the exponent $\beta$ changes very little with $\lambda$ for $\sigma=1.5$ and 3.0 for each of which $(\lambda, \sigma)$ combinations correspond to the same $P_{1}, P_{2}$ and $P_{3}$ phases with DL edges. This happens possibly because the properties of the localized states barely vary in the different $P_{q}$ phases. On the other hand $\beta$ changes rapidly with $\lambda$ for $\sigma=0.5$ in the presence of multifractal states the properties of which may change significantly as one moves from $P_{1}$ to $P_{2}$ to $P_{3}$ and so on. Another observation is that the late time dynamics of $S_{\mathrm{A}}$ slows down for $\sigma=1.5$ whereas it speeds up for $\sigma=3.0$. This happens due to varying degrees of effectiveness of the delocalized eigenstates in the presence of long-range and short-range hopping. In a particular $P_{q}$ phase (with DM or DL edges) the values of all the exponents discussed here barely change with $\lambda$ for a fixed value of $\sigma$. Similar results have been discussed in a recent work [68]. Also we have checked that the qualitative behaviors of all the $S_{\mathrm{A}}$ plots and the values of the exponent $\beta$ change very little if, instead of $\alpha_{\mathrm{g}}$, one uses $\alpha_{\mathrm{s}}$ or $\alpha_{\mathrm{b}}$ for an initial half filled CDW state. However, the exponents associated with the secondary $S_{\mathrm{A}}$ growth may change significantly as this part of the dynamics is dominated by the multifractal or 


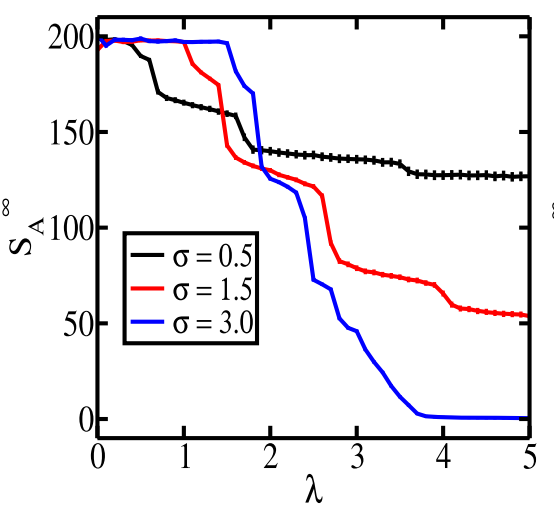

(a)

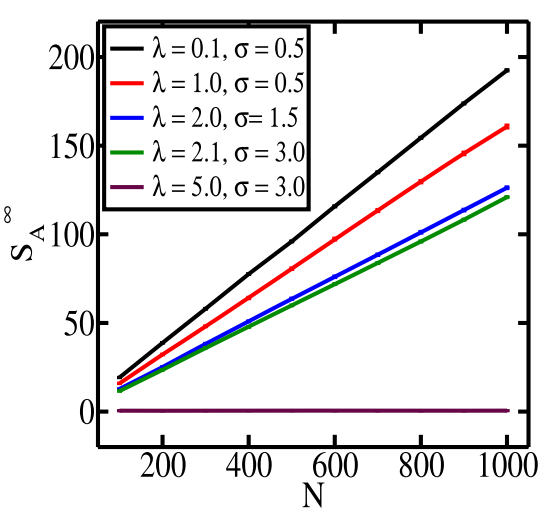

(b)

Figure 4. (a) The saturation value of the half-system $S_{\mathrm{A}}$ as a function of $\lambda$ for $\sigma=0.5,1.5$ and 3.0 respectively for fermions at half-filling. (b) The system size $N$ dependence of the saturation value of the half-chain entanglement entropy $S_{\mathrm{A}}^{\infty}$ of free fermions at half-filling for different combinations of $\lambda$ and $\sigma$.

the localized single particle eigenstates, the fraction of which depends on the choice of the quasiperiodicity parameter in a particular $P_{q}(q=1,2,3, \ldots)$ phase.

The saturation value of entanglement entropy $S_{\mathrm{A}}^{\infty}$ turns out to be a useful quantity. Figure 4(a) shows $S_{\mathrm{A}}^{\infty}$ as a function of $\lambda$ for $\sigma=0.5,1.5,3.0$. The steps appearing in the plots denote the transitions from the delocalized-to $P_{1}$-to- $P_{2}$-to- $P_{3}$ etc phases. The $P_{q}$ phases have a fraction of eigenstates that are multifractal for $\sigma=0.5$ and a fraction of eigenstates that are localized for $\sigma=1.5,3.0$. Hence $S_{\mathrm{A}}^{\infty}$ is much lower for $\sigma=1.5,3.0$ than for $\sigma=0.5$ in these phases. Also we have looked at the system size $N$ dependence of $S_{\mathrm{A}}^{\infty}$ in these phases as shown in figure $4(\mathrm{~b})$. The combinations of $(\lambda, \sigma)$ are chosen in such a way that the system is in the delocalized phase for $(0.1,0.5) ; P_{2}$ phase with DM edge for $(1.0,0.5) ; P_{2}$ phase with DL edge for $(2.0,1.5)$, and $(2.1,3.0)$; and localized phase for $(5.0,3.0)$. For the delocalized and mixed phases with DM or DL edges $S_{\mathrm{A}}^{\infty} \propto N$. In the localized phase $S_{\mathrm{A}}^{\infty} \propto N^{0}$, which is obtained effectively in the short-range AAH limit. Typically in a sufficiently long-ranged regime one can obtain algebraic localization such as seen in the random long-range hopping model [63]. In the random long-range hopping model an algebraic localization dominated phase is found for $1<\sigma<2$ for which $S_{\mathrm{A}}^{\infty}$ varies sub-linearly with $N$ [63].

\section{Out-of-time-order correlator}

OTOC are good observables to capture chaos or information scrambling in quantum systems. The majority of studies looking at OTOC have been in the context of localization transitions in interacting systems [13, 15-17, 46]. However, OTOC has been barely $[53,54]$ addressed in the literature in relation to the localization transition in disordered noninteracting (quadratic) Hamiltonians. Our goal here is to investigate OTOC as a distinguisher for the various phases found in the AAH and LRH models. In this work we choose the two unitary-and-Hermitian operators $\hat{\sigma}_{i}^{z}$ and $\hat{\sigma}_{j}^{z}$ at a distance $x=|i-j|$. The function $F(x, t)$ in equation (2) is then given by

$$
F(x, t)=\left\langle\hat{\sigma}_{i}^{z}(t) \hat{\sigma}_{j}^{z}(0) \hat{\sigma}_{i}^{z}(t) \hat{\sigma}_{j}^{z}(0)\right\rangle .
$$

We keep the position of the time evolved operator fixed at $i=N / 2$. By varying $j$ we study the scrambling of quantum information over the lattice as a function of time. The initial state is fixed as the product state of half-filled fermions defined in equation (4). For free fermions one can use the Jordan-Wigner transformation $\hat{\sigma}_{j}^{z}=2 \hat{n}_{j}-1$ to simplify the expression of $F(x, t)$ [53]. We elaborate on this ahead.

\subsection{Formalism}

Here we provide a brief description of the formalism in relation to OTOC which is used in this work. Let us consider a generic quadratic Hamiltonian:

$$
\hat{H}_{\text {free }}=\sum_{i, j} H_{i j} \hat{c}_{i}^{\dagger} \hat{c}_{j},
$$

where $H_{i j}$ 's are the elements of a Hermitian matrix $H$ and $\hat{c}_{i}^{\dagger}$ 's $\left(\hat{c}_{i}\right.$ 's) are fermionic creation (annihilation) operators obeying the following anti-commutation relations:

$$
\left\{\hat{c}_{i}^{\dagger}, \hat{c}_{j}\right\}=\delta_{i j} ;\left\{\hat{c}_{i}^{\dagger}, \hat{c}_{j}^{\dagger}\right\}=\left\{\hat{c}_{i}, \hat{c}_{j}\right\}=0
$$

Using the eigenvectors of the coupling matrix $H$, we can define new fermionic operators that diagonalize the Hamiltonian. If $A_{j k}$ represent the coefficients of the eigenvectors of the matrix $H$, we introduce the fermionic operators:

$$
\hat{d}_{k}^{\dagger}=\sum_{j} A_{j k}^{*} \hat{c}_{j}^{\dagger}, \quad \hat{d}_{k}=\sum_{j} A_{j k} \hat{c}_{j}
$$

that transform the Hamiltonian into a diagonal form:

$$
\hat{H}_{\text {free }}=\sum_{k} \epsilon_{k} \hat{d}_{k}^{\dagger} \hat{d}_{k}
$$

Here $\hat{d}_{k}^{\dagger}\left(\hat{d}_{k}\right)$ creates (annihilates) a particle with energy $\epsilon_{k}$ and obeys similar anti-commutation relations as $\hat{c}_{i}$ 's:

$$
\left\{\hat{d}_{k}^{\dagger}, \hat{d}_{l}\right\}=\delta_{k l},\left\{\hat{d}_{k}, \hat{d}_{l}\right\}=0,\left\{\hat{d}_{k}^{\dagger}, \hat{d}_{l}^{\dagger}\right\}=0 .
$$


Using the Heisenberg equation for operators, the time-evolved operators $\hat{d}_{k}^{\dagger}(t)$ and $\hat{d}_{k}(t)$ can be found.

$$
\frac{\mathrm{d}}{\mathrm{d} t} \hat{d}_{k}=\imath\left[\hat{H}_{\mathrm{free}}, \hat{d}_{k}\right]=-\imath \epsilon_{k} \hat{d}_{k}
$$

which leads to

$$
\hat{d}_{k}(t)=\mathrm{e}^{-\imath \epsilon_{k} t} \hat{d}_{k}(t=0)
$$

and hence $\hat{d}_{k}^{\dagger}(t)=\mathrm{e}^{\imath \epsilon_{k} t} \hat{d}_{k}^{\dagger}(t=0)$. Using the relations:

$$
\hat{c}_{j}^{\dagger}(t)=\sum_{k} A_{j k} \hat{d}_{k}^{\dagger}(t), \quad \hat{c}_{j}(t)=\sum_{k} A_{j k}^{*} \hat{d}_{k}(t)
$$

one finds the following anti-commutation relations between creation and annihilation operators at different times in position space.

$$
\begin{aligned}
& \left\{\hat{c}_{i}^{\dagger}(t), \hat{c}_{j}\right\}=\sum_{k} \mathrm{e}^{\imath \epsilon_{k} t} A_{i k}^{*} A_{j k}=a_{i j}(t) \\
& \left\{\hat{c}_{i}(t), \hat{c}_{j}^{\dagger}\right\}=\sum_{k} \mathrm{e}^{-\imath \epsilon_{k} t} A_{i k} A_{j k}^{*}=a_{i j}^{*}(t)
\end{aligned}
$$

along with $\left\{\hat{c}_{i}^{\dagger}(t), \hat{c}_{j}^{\dagger}\right\}=\left\{\hat{c}_{i}(t), \hat{c}_{j}\right\}=0$, which are trivially satisfied. Here the parentheses used to denote time are dropped from the operators for $t=0$. This convention is used further in the paper.

In this work we consider an initial product state of the form

$$
|\Psi\rangle=\prod_{j \in S} \hat{c}_{j}^{\dagger}|0\rangle
$$

where $j$ refers to the index of the site which is occupied. Let $S$ be the set consisting of site indices of sites which are occupied. The initial occupation matrix in position space is then given by

$$
\left\langle c_{i}^{\dagger} \hat{c}_{j}\right\rangle= \begin{cases}1 & \text { if } i=j \quad \forall i \in S \\ 0 & \text { otherwise. }\end{cases}
$$

Using the Jordan-Wigner transformation $\hat{\sigma}_{i}^{z}=2 \hat{n}_{i}-1$ with $\hat{n}_{i}=\hat{c}_{i}^{\dagger} \hat{c}_{i}$ in equation (5) we have:

$$
\begin{aligned}
F(x, t)= & 16\left\langle\hat{n}_{i}(t) \hat{n}_{j} \hat{n}_{i}(t) \hat{n}_{j}\right\rangle+4\left\langle\hat{n}_{j} \hat{n}_{i}(t)\right\rangle-4\left\langle\hat{n}_{i}(t) \hat{n}_{j}\right\rangle \\
& -8\left\langle\hat{n}_{i}(t) \hat{n}_{j} \hat{n}_{i}(t)\right\rangle-8\left\langle\hat{n}_{j} \hat{n}_{i}(t) \hat{n}_{j}\right\rangle+1 .
\end{aligned}
$$

In this work we have kept $i=N / 2$ where $N$ is the number of sites in the lattice and calculated $F(x, t)$ by varying $j$. For the case $j \in S$ such that $\hat{c}_{j}^{\dagger}|\Psi\rangle=0$, equation (17) can be written as [53]

$$
F(x, t)=8\left|a_{i j}\right|^{2}\left\langle\hat{n}_{i}(t)\right\rangle-8\left|a_{i j}\right|^{2}+1 .
$$

For $j \notin S, \hat{c}_{j}|\Psi\rangle=0$ which leads to

$$
F(x, t)=1-8\left|a_{i j}\right|^{2}\left\langle\hat{n}_{i}(t)\right\rangle
$$

\subsection{Results}

We now discuss the OTOC-related results for the AAH and LRH models.

$A A H$ model: first we calculate $C(x, t)$ in the AAH model. The profiles of $C(x, t)$ in position space for increasing instants of time are shown in figures 5(a)-(c) for $\lambda=1.0,2.0$ and 3.0 respectively. At $t=0 C(x)$ is zero for all $x$ because $F(x, 0)$ reduces to the squares of Pauli matrices yielding unity in equation (5). Then $C(x)$ starts developing for small values of the distance $x$ due to the non-commutation of the matrices $\hat{\sigma}_{i}^{z}(t)$ and $\hat{\sigma}_{j}^{z}(0)$ for small $x$ at early times. During this period of time $C(x)$ attains high values for small $x$ while the maximum value of $C(x)$ happens to be at $x=1$. This is shown in figure 5(a) for $\lambda=1$. Then $C(x, t)$ starts decreasing for small $x$ whereas it keeps growing for large values of $x$ due to the spreading of noncommutativity among Pauli matrices. In the long run $C(x, t)$ shows a uniform dependence on $x$ for $\lambda=1$ (see figure 5(a)) when $S_{\mathrm{A}}$ also reaches saturation. For the critical point $\lambda=2$ the initial dynamics of $C(x, t)$ shown in figure $5(\mathrm{~b})$ is similar to that for $\lambda=1$. But in the long-time limit $C(x, t)$ shows a non-uniform dependence on $x$ with occasionally large fluctuations especially at $x=21,34,55$ etc which are terms in the Fibonacci sequence of the 'golden mean' [69]. These large fluctuations appear possibly due to the multifractal nature of the eigenstates. In figure 5(c) for the localized phase at $\lambda=3$, $C(x, t)$ grows for small $x$ at early times while the subsequent decay is absent in the dynamics. Eventually in the long-time limit $C(x)$ drops exponentially with $x$ i.e. $C(x) \sim \mathrm{e}^{-x / \xi_{\text {Отос }}}$ [53] such that $C(x) \neq 0$ for $x<\xi_{\text {Oтос }}$ but is zero for large $x$. $\xi_{\text {OтоC }}$ decreases with $\lambda$ in the localized phase.

Also we analyse the system size $N$-dependence of the spatial profile of $C^{\infty}(x)$ in the long-time limit as shown in figures 5(d)-(f) for $\lambda=1,2,3$ respectively. For $\lambda=1, C^{\infty} \propto$ $1 / N$. This can be explained by looking at the long-time behavior of $\left|a_{i j}(t)\right|^{2}$ defined in section 4.1. $\lim _{T \rightarrow \infty} \frac{1}{T} \int_{0}^{T} \mathrm{~d} t\left|a_{i j}(t)\right|^{2}=$ $\sum_{k}\left|A_{i k}\right|^{2}\left|A_{j k}\right|^{2}$, which scales with $1 / N$ as $A_{i k} \propto 1 / \sqrt{N}$ in the delocalized phase. At the critical point $\lambda=2, C^{\infty}$ depends on $x$ and shows a sub-linearly decreasing dependence with $N$ except on the points where large fluctuations are observed due to the multifractal nature of the eigenstates. At these special points the $N$-dependence is not regular. The number of these large fluctuations increases with $N$. However, in the localized phase for $\lambda=3, C^{\infty} \propto N^{0}$ for $x<\xi_{\text {отос }}$ and is in any case zero for large $x$.

The early-time growth of OTOC in the AAH model is shown in figures 6(a)-(c) for small values of $x$ and for $\lambda=1$, 2,3 respectively. For all values of $\lambda$ we notice that $C(x, t) \sim$ $t^{2 x} \forall$ odd $x$ and $C(x, t) \sim t^{2(x+1)} \forall$ even $x$, which is also found in translationally invariant models [49]. This can be understood by writing the Heisenberg time evolution of $\hat{W}(t)$ using the Hausdorff-Baker-Campbell formula

$$
\mathrm{e}^{\mathrm{i} t \hat{H}} \hat{W} \mathrm{e}^{-\mathrm{i} t \hat{H}}=\sum_{m=0}^{\infty} \frac{(\mathrm{i} t)^{m}}{m !} \hat{L}^{m}(\hat{W}),
$$

where $\hat{L}(\hat{W})=[\hat{H}, \hat{W}]$ and $\hat{W}=\hat{\sigma}_{L / 2}^{z}$. The power-law growth obtained in the early-time dynamics is controlled by the term 


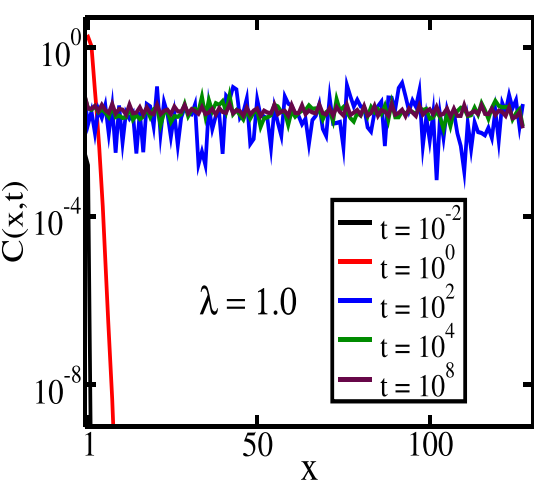

(a)

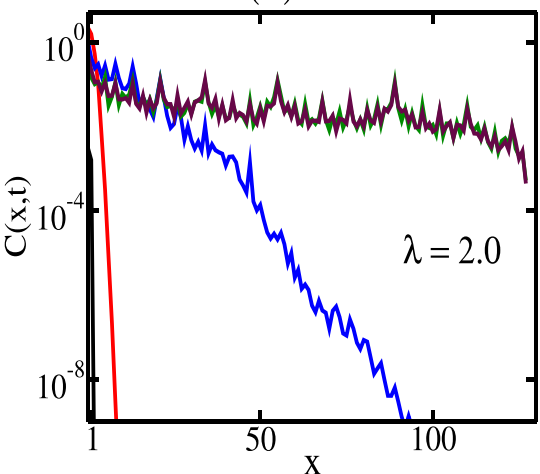

(b)

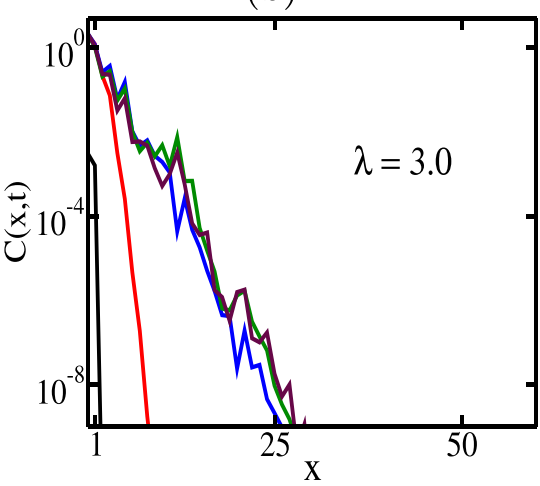

(c)

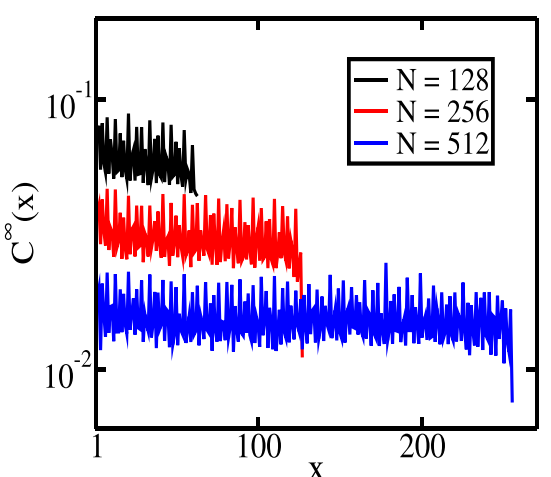

(d)

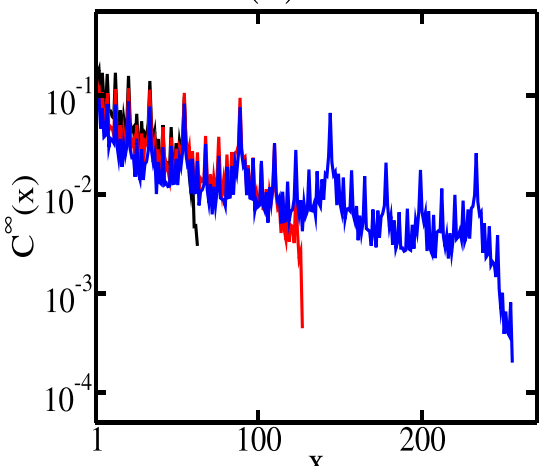

(e)

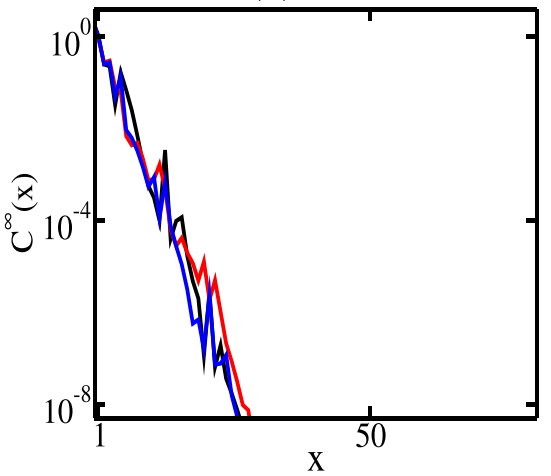

(f)

Figure 5. OTOC in the AAH model. (a)-(c) OTOC $C(x, t)$ as a function of distance $x$ at different instants $t$ for $\lambda=1.0,2.0$ and 3.0 respectively. System size $N=256$. The plot legend shown in (a) also applies to (b) and (c). (d)-(f) Saturation value $C^{\infty}(x)$ as a function of distance $x$ for increasing system sizes $N$ and for $\lambda=1.0,2.0$ and 3.0 respectively. The plot legend shown in (d) also applies to (e) and (f). For all the plots, total number of $\theta_{\mathrm{p}}$ realizations is 500 .

with the smallest $m$ such that $\left.\left.\left[\hat{L}^{m}, \hat{\sigma}_{L / 2+x}^{z}\right]\right)\right] \neq 0$. For shortrange AAH Hamiltonian it is clear that this happens when $m=x$ leading to $C(x, t) \sim t^{2 x}[49]$. For $x=2,4,6, \ldots$ one includes the next leading term which gives $C(x, t) \sim t^{2(x+1)}$ [53]. This shows that the quasiperiodic disorder does not play any important role in the initial dynamics. However, in the long-time limit OTOC is found to decay as $1 / t^{\gamma}$ with time and the power-law exponent $\gamma$ depends on $\lambda$ as shown in figures 6 (d)-(f) for $\lambda=1,2,3$ respectively. We find that the values of $\gamma \approx 1.0,0.3,0.0$ for $\lambda=1,2,3$ respectively which correspond to the delocalized, critical and localized phases respectively. The $t^{-1}$ decay in the delocalized phase is also seen in a clean system [49]. The extended (ergodic or nonergodic) states are responsible for the correlation wavefront to reach a particular distant site in the lattice (leading to OTOC growth) and then proceed further (leading to OTOC decay) until OTOC reaches saturation. The decay rate is expectedly less in the (nonergodic) multifractal phase in comparison to the (ergodic) delocalized phase. A lot of intrinsic fluctuations are found in these plots due to the presence of quasiperiodic disorder. We also note that in the late time dynamics for a fixed value of $\lambda$ the value of $\gamma$ does not depend on $x$ unlike the early-time growth.

LRH model: the spatial distribution of OTOC for the LRH model of fermions at half-filling is shown in figure 7 . We have chosen the combination of parameters $(\lambda, \sigma)$ in such a way that 


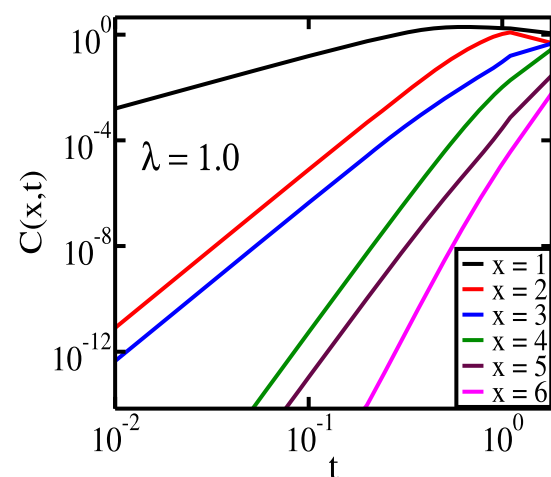

(a)

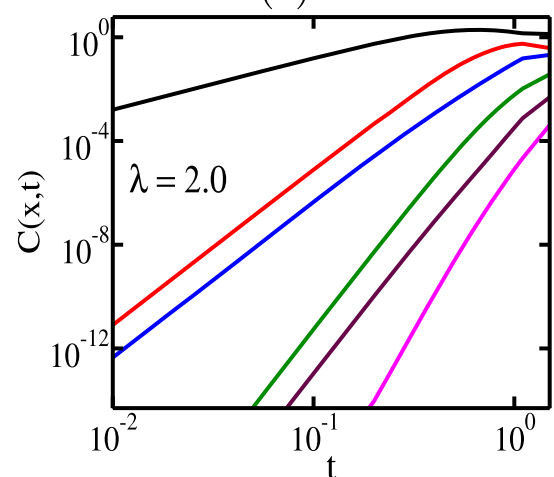

(b)

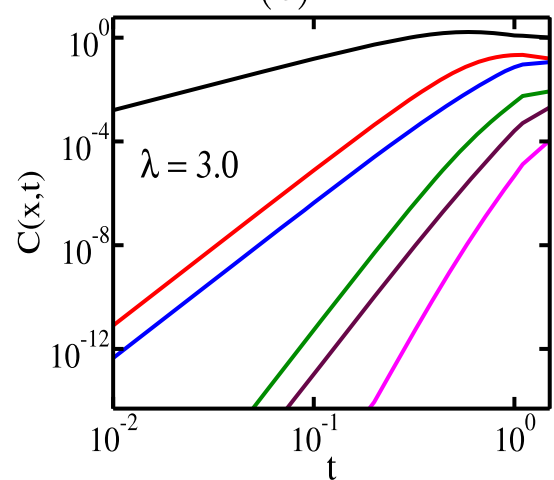

(c)

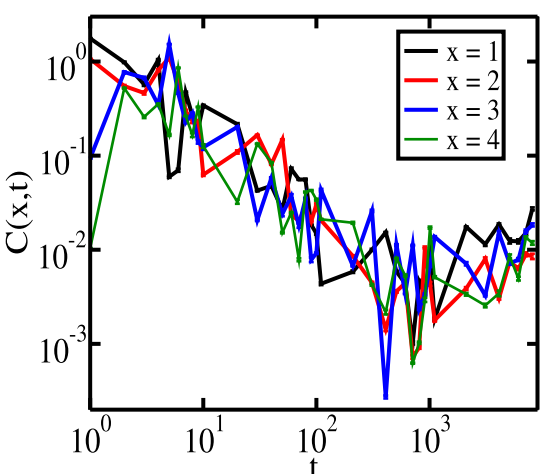

(d)

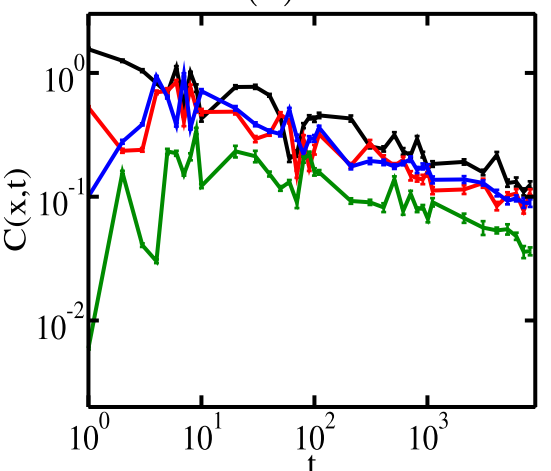

(e)

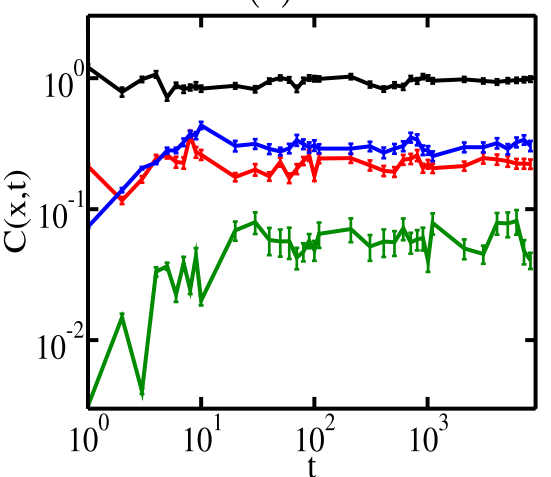

(f)

Figure 6. Time dynamics of OTOC in the AAH model for increasing values of $x$. (a) - (c) $C(x, t)$ vs $t$ plots for early times for $\lambda=1.0,2.0$ and 3.0 respectively. The plot legend shown in (a) also applies to (b) and (c). (d)-(f) $C(x, t)$ vs $t$ plots for late times for $\lambda=1.0,2.0$ and 3.0 respectively. The plot legend shown in (d) also applies to (e) and (f). For all the plots, system size $N=1024$ and total number of $\theta_{\mathrm{p}}$ realizations is 500 .

the system is in four different types of phases: (i) $P_{2}$ phase with DM edge $(\sigma=0.5)$, (ii) $P_{2}$ phase with DL edge where the hopping is relatively long-range $(\sigma=1.5)$, (iii) $P_{2}$ phase with DL edge where the hopping is short-range $(\sigma=3.0)$ and (iv) the localized phase. The spatial profiles of $C(x, t)$ for each of the above kinds of parameter combinations are shown in figures $7(\mathrm{a})-(\mathrm{d})$ respectively. For early times $C(x, t)$ shows $1 / x^{2 \sigma}$ dependence for all the choices of parameters.

In the mixed phases we see that in the long time limit $C(x, t)$ follows $1 / x^{\delta}$ (power-law) behavior for small $x$ and almost $x$ independent behavior for large $x$. In figure 7 (a) $\delta \approx 1.0$ for small $x$ whereas occasional large fluctuations can be seen for large values of $x$ which are terms in the Fibonacci sequence of the 'golden mean'. The occasional large fluctuations are signatures of the multifractal states similar to the AAH model at the transition point. In figure 7(b) for intermediate times $(t \sim 10) C(x, t)$ shows $1 / x^{1.5}$ dependence for small $x$ and $1 / x^{2 \sigma}$ dependence for large $x$. However, in the long time limit $x$ independent behavior of $C(x, t)$ is seen for large $x$ along with the $1 / x^{1.5}$ dependence for small $x$. Here $\delta \approx 1.5$. In figure 7(c) for intermediate times $C(x, t)$ shows $1 / x^{1.5}$ dependence for small $x$ and $1 / x^{2 \sigma}$ dependence for large $x$. A sharp boundary can be seen between these two behaviors, which is a characteristic signature of the short-range regime [43]. In the long time limit $C(x, t)$ does not depend on $x$ for large $x$ whereas it continues to show the $1 / x^{1.5}$ dependence for small $x$ corresponding to $\delta \approx 1.5$ once again. In figure $7(\mathrm{~d})$ corresponding to the localized phase the spatial profile $C(x, t)$ continues to be $1 / x^{2 \sigma} \forall x$. We do not a see a 'mixed' behavior in this case as the system is unambiguously in the localized phase. We notice that 


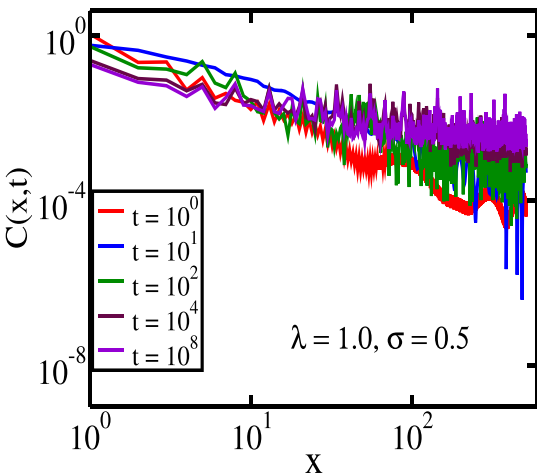

(a)

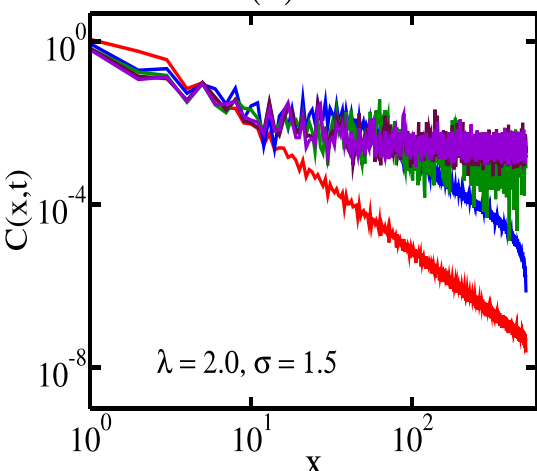

(b)

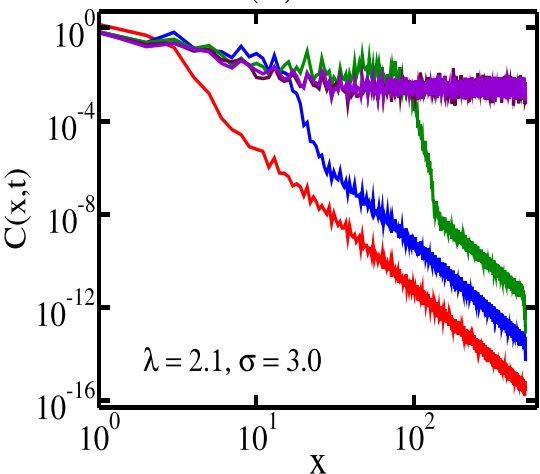

(c)

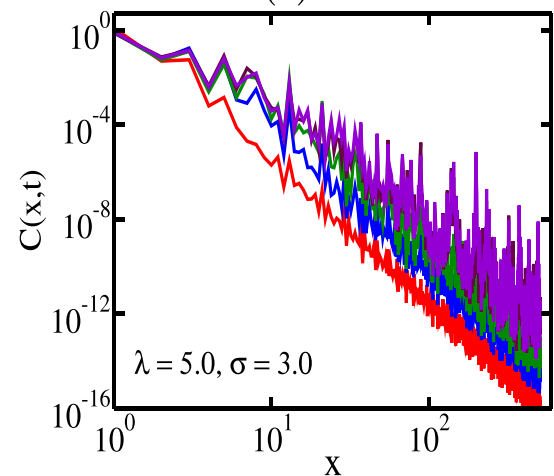

(d)

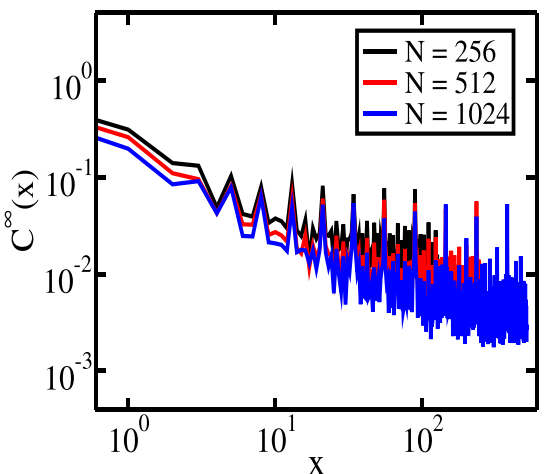

(e)

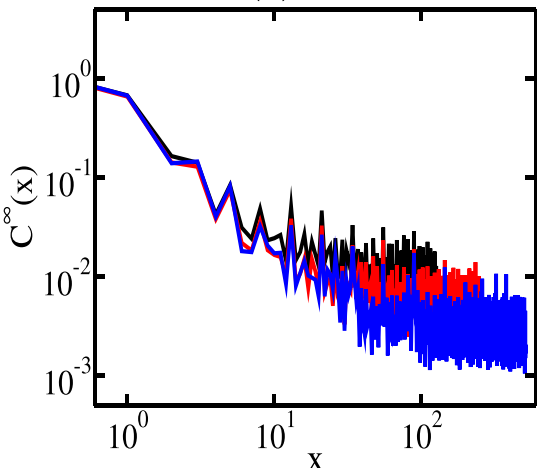

(f)

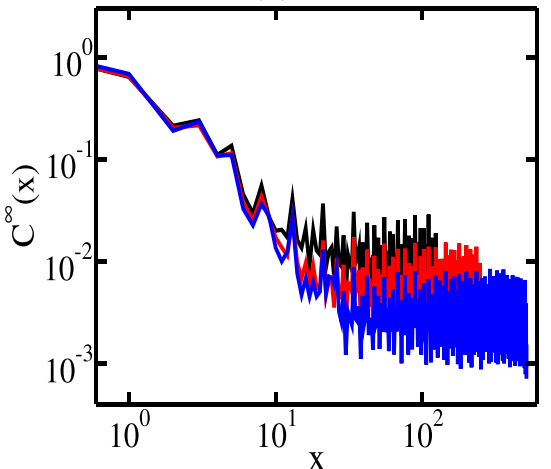

(g)

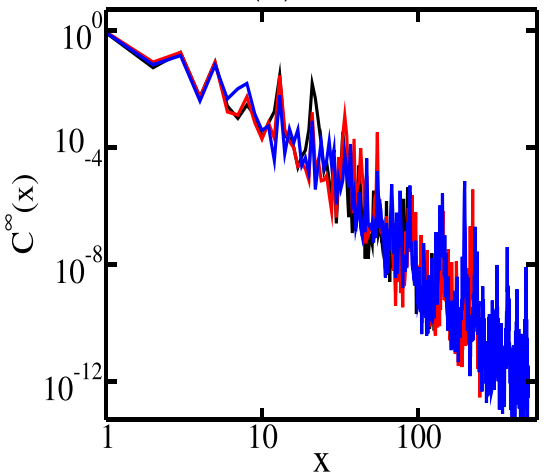

(h)

Figure 7. OTOC in the LRH model. (a)-(d) OTOC $C(x, t)$ as a function of distance $x$ at different instants $t$ for $(\lambda=1.0, \sigma=$ $0.5),(\lambda=2.0, \sigma=1.5),(\lambda=2.1, \sigma=3.0)$ and $(\lambda=5.0, \sigma=3.0)$ respectively. System size $N=1024$ the plot legend shown in (a) also applies to (b), (c) and (d). (e)-(h) Saturation value $C^{\infty}(x)$ as a function of distance $x$ for increasing system sizes $N$ and for $(\lambda=1.0, \sigma=0.5),(\lambda=2.0, \sigma=1.5),(\lambda=2.1, \sigma=3.0)$ and $(\lambda=5.0, \sigma=3.0)$ respectively. The plot legend shown in figure (e) also applies to figures (f), (g) and (h). For all the plots, total number of $\theta_{\mathrm{p}}$ realizations is 500 . 


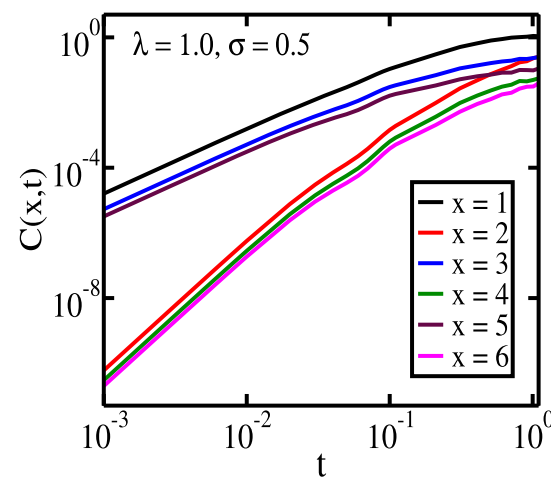

(a)

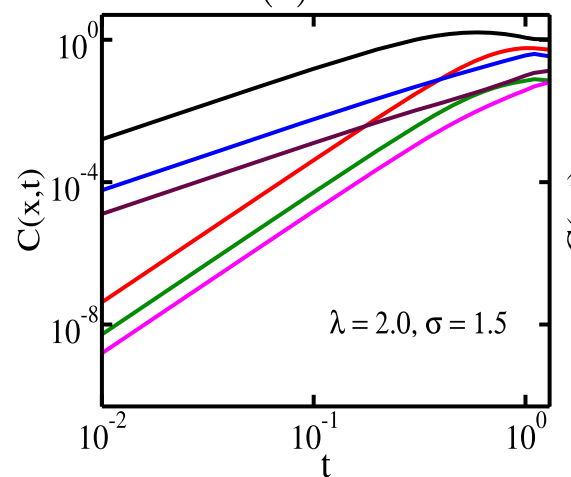

(b)

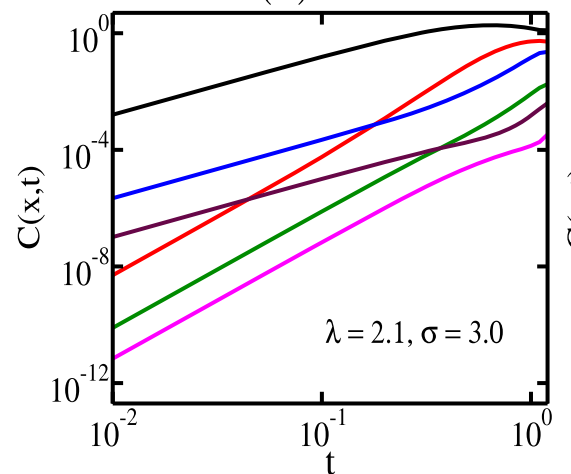

(c)

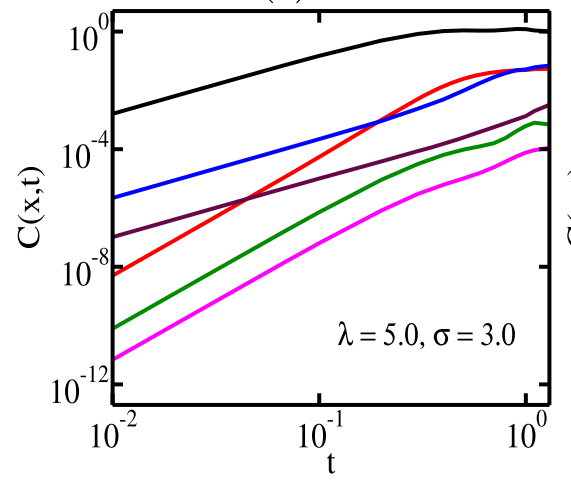

(d)

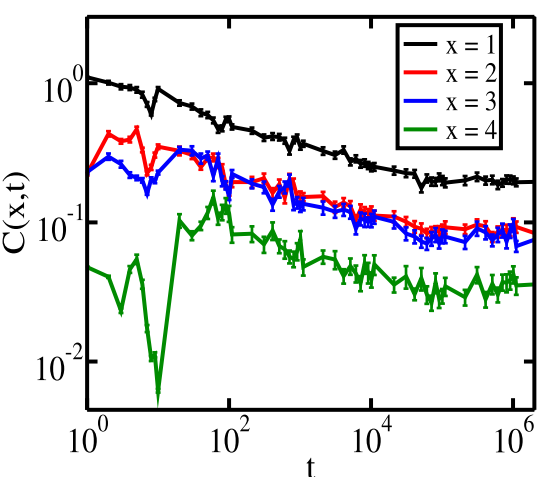

(e)

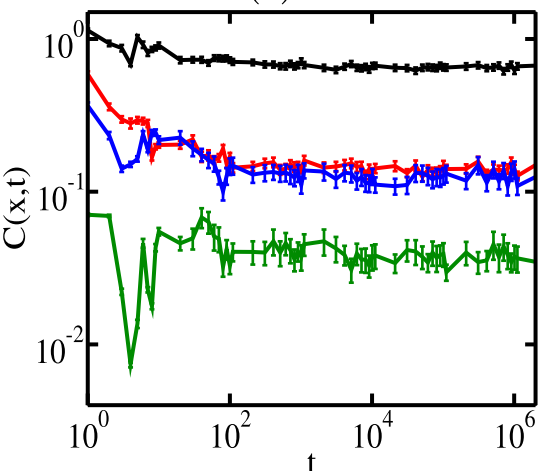

(f)

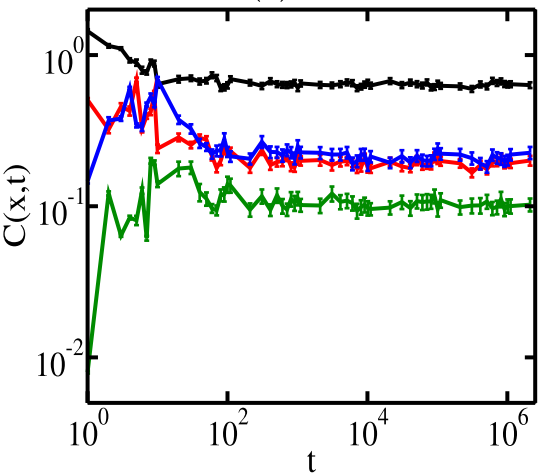

(g)

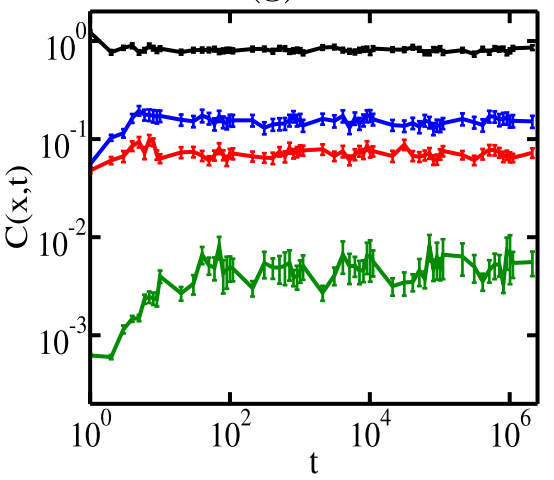

(h)

Figure 8. Time dynamics of OTOC in the LRH model for increasing values of $x$. (a)-(d) $C(x, t)$ vs $t$ plots for early times for $(\lambda=1.0, \sigma=0.5)$, $(\lambda=2.0, \sigma=1.5),(\lambda=2.1, \sigma=3.0)$ and $(\lambda=5.0, \sigma=3.0)$ respectively. The plot legend shown in (a) also applies to (b), (c) and (d). (e) $-(\mathrm{h})$ $C(x, t)$ vs $t$ plots for late times for $(\lambda=1.0, \sigma=0.5),(\lambda=2.0, \sigma=1.5),(\lambda=2.1, \sigma=3.0)$ and $(\lambda=5.0, \sigma=3.0)$ respectively. The plot legend shown in figure (e) also applies to figures (f), (g) and (h). For all the plots, system size $N=1024$ and total number of $\theta_{\mathrm{p}}$ realizations is 500 . 
the value of $\delta \approx 1.0$ for all the mixed phases with DM edges $(\sigma<1)$ while $\delta \approx 1.5$ for the mixed phases with DL edges $(\sigma>1)$. It is noticeable that the value of $\delta$ is larger in the presence of localized states than that in the presence of multifractal states.

In figures 7(e)-(h) we show the system size dependence of the spatial profile of OTOC in the long time limit $C^{\infty}(x)$ corresponding to phases described in figures $7(\mathrm{a})-(\mathrm{d})$ respectively. Since we have already mentioned earlier that the calculation of $C^{\infty}(x)$ involves each eigenstate in the spectrum, in the mixed phases $\left(P_{2}\right.$ phase with DL/DM edge), in figures 7(e)-(g) $C^{\infty}(x)$ for large $x$ decreases with the system size $N$ although its functional dependence on $N$ is not very clear due to fluctuations. In figure 7(e) we see the occasional large fluctuations due to the presence of multifractal states increase and become more prominent with $N$. In figures 7(f) and (g) the large fluctuations are not seen due to the absence of multifractal states. In the presence of DM edge $C(x)$ depends on $N$ for small $x$ whereas in the presence of DL edge $C(x)$ remains invariant with the change in $N$ for small $x$. In figure 7(h) the spatial profile of $C^{\infty}(x)$ becomes system size independent which is characteristic of a localized phase. We note that in the presence of localized states the peak of the profile of $C^{\infty}(x)$ has a higher value (figures $7(f)-(h)$ ) than in the absence of localized states (figure 7(e)).

The early-time growth of OTOC in the LRH model is shown for small $x$ in figure 8 for the $P_{2}$ phase with DM edge in figure $8(\mathrm{a}), P_{2}$ phase with DL edge in figures $8(\mathrm{~b})-(\mathrm{c})$ and the localized phase in figure $8(\mathrm{~d})$. Independent of the values of $\lambda$ and $\sigma$, we find that $C(x, t) \sim t^{2} \forall$ odd $x$ and $C(x, t) \sim t^{4} \forall$ even $x$ as also found for the translationally invariant long-range hopping model (we have checked). Unlike the short-range AAH model here OTOC does not have a power-law behavior with $x$ and the growth here is in fact largely $x$-independent. This can be again understood from equation (20). Since the LRH Hamiltonian is long-ranged $\hat{\sigma}_{L / 2}^{z}$ and $\hat{\sigma}_{L / 2+x}^{z}$ immediately get connected for smallest $m=1$ which gives $C(x, t) \sim t^{2}$ [70] for odd $x$. For even $x$, including the next-to-leading-order term, we obtain $C(x, t) \sim t^{4}$. For the same phases of the LRH model, the late-time decay of OTOC is shown in figures $8(\mathrm{e})-(\mathrm{h})$. From figure 8(e) we see that in the $P_{2}$ phase with DM edge the power-law decay exponent $\gamma=0.15 \forall x$. In figures 8(f)-(g) we find that in the $P_{2}$ phase with DL edge the decay exponent gets smaller, and is difficult to determine. A power-law decay is found due to the presence of the delocalized states in the phase. However it is smaller as compared to that in figure 8(e) due to the presence of localized states instead of (extended nonergodic) multifractal states. In figure 8(h) we do not see any decay of OTOC after the early-time growth due to the absence of delocalized or multifractal states. We have checked that all the dynamical behaviors shown by both the entanglement entropy and OTOC can be seen more clearly as the system size is increased.

We would also like to mention that we have checked that for a clean (undisordered) system in the presence of long-range hopping, in the long-time limit $C(x, t)$ decays as $1 / t$ independent of $x$ and the long-range parameter $\sigma$. The values of $C^{\infty}$ are also independent of $x$ and $\sigma$ as the phases are delocalized for all $\sigma$. On the other hand in the LRH model, the power-law decay exponent $\gamma$ is much lower than that in the clean system. In the LRH model $\gamma$ depends on the values of $\sigma$. The values of $C^{\infty}$ depend on $x$ as the phases are (nonergodic) mixed or localized. However, the values of $\gamma$ and $C^{\infty}$ change very little with the fraction of delocalized states present in the mixed phases, especially in the presence of the DM edge.

\section{Conclusion}

To conclude we study the nonequilibrium dynamics of entanglement entropy and OTOC of noninteracting fermions at half-filling starting from a product state to distinguish different phases hosted by the quasiperiodic AAH model with long-range hopping. Apart from the delocalized and localized phases, the model also shows mixed phases which consist of delocalized and multifractal or localized states. In the nearest neighbor hopping limit due to the restoration of self-duality the model hosts delocalized, multifractal and localized phases. When the hopping is sufficiently long-ranged a secondary logarithmic behavior in the entanglement entropy is seen in the mixed phases whereas the primary behavior is a power-law growth which can be different in different phases. The saturation value of entanglement entropy in the delocalized, multifractal and mixed phases depends linearly on system size whereas in the localized phase (in the short-range regime) it is independent of system size. The secondary growth is a unique feature that we expect to see in the long-ranged mixed phases of other models as well, since this feature seems to be connected to the long-ranged nature of the hopping. Although in our specific system, we find that the secondary growth is logarithmic, this may not necessarily be true in other long-ranged systems. In early-time dynamics OTOC shows very different behavior in the presence of nearest neighbor hopping and longrange hopping, like is seen also in clean systems. The late-time decay rate of OTOC is different in the delocalized and multifractal phases of the nearest neighbor AAH model whereas the localized phase of the same model shows no such decay. In the long-time limit the spatial profile of OTOC is independent, dependent (with large fluctuations) and exponentially dependent on space in the delocalized, multifractal and localized phases respectively. Also the profile decreases linearly and sub-linearly with system size in the delocalized and multifractal phases respectively whereas it is independent of system size in the localized phase. In the multifractal phase, large fluctuations are observed at the special points which are related to the Fibonacci sequence of the quasiperiodicity parameter. In the LRH model, the late-time power-law decay is present in the mixed phases due to the presence of extended states although the power-law decay exponent is smaller compared to the inverse power-law behavior found in the delocalized phase of the (clean) system. The power-law exponent barely changes with the change in the fraction of delocalized states in the mixed phases showing the dominance of the nonergodic states in the dynamics. In the mixed phases the presence of localized states suppresses the late-time decay even more than multifractal states. The localized phase of this model does not 
show any such decay due to absence of extended states. The dynamics of the spatial profile of OTOC in the mixed phase in the short-range limit reveals a sharp boundary which is typical of longrange models [67]. The spatial profile of OTOC in the long-time limit in the mixed phases shows a mixed behavior: power-law dependence for small distance (nonergodic behavior) and no dependence for large distance (ergodic behavior). In the mixed phases containing multifractal states the profile shows large fluctuations at special points for large distance similar to the critical point of the AAH model. In the localized phase the spatial dependence of OTOC is a power-law one for all distances and is also independent of system size. Also in the mixed phases the spatial profile shows different system-size dependences for small and large distances which is expected. One may expect to see these behaviors in the mixed phases of other long-range (Harper-like) models.

Entanglement entropy and OTOC are two quantities that are of great interest in dynamical studies of quantum systems with the second one being easier to be implemented in experiments. Very recently, a surge of interest in the community [71-74] has been seen in the experimental detection of quantum phase transitions using OTOC. At this point our work provides the temporal and spatial features of OTOC to detect a host of different quantum phases which can potentially be implemented in the ongoing experiments. Also there are possibilities of studying the temperature dependence of OTOC in the LRH model using a thermal state which one can address in the future.

\section{Acknowledgments}

NR would like to acknowledge University Grants Commision (UGC), India for providing a $\mathrm{PhD}$ fellowship and thanks Kamanpreet Singh Manoor for fruitful discussions on OTOC. AS acknowledges financial support from SERB via the Grant (File No.: CRG/2019/003447), and from DST via the DSTINSPIRE Faculty Award [DST/INSPIRE/04/2014/002461].

\section{Data availability statement}

The data generated and/or analysed during the current study are not publicly available for legal/ethical reasons but are available from the corresponding author on reasonable request.

\section{ORCID iDs}

Nilanjan Roy (D) https://orcid.org/0000-0003-3016-7146

Auditya Sharma (D) https://orcid.org/0000-0002-1124-9528

\section{References}

[1] Laflorencie N 2016 Phys. Rep. 6461

[2] Eisert J, Cramer M and Plenio M B 2010 Rev. Mod. Phys. 82 277

[3] Vidal G, Latorre J I, Rico E and Kitaev A 2003 Phys. Rev. Lett. 90227902
[4] Serbyn M, Papić Z and Abanin D A 2013 Phys. Rev. Lett. 110 260601

[5] Alet F and Laflorencie N 2018 C. R. Physique 19498

[6] Abanin D A, Altman E, Bloch I and Serbyn M 2019 Rev. Mod. Phys. 91021001

[7] Hashimoto K, Murata K and Yoshii R 2017 J. High Energy Phys. JHEP10(2017)138

[8] Shenker S H and Stanford D 2015 J. High Energy Phys. JHEP05(2015)132

[9] Maldacena J, Shenker S H and Stanford D 2016 J. High Energy Phys. JHEP08(2016)106

[10] Roberts D A and Swingle B 2016 Phys. Rev. Lett. 117 091602

[11] Roberts D A and Stanford D 2014 arXiv:1412.5123

[12] Swingle B 2018 Nat. Phys. 14988

[13] Swingle B and Chowdhury D 2017 Phys. Rev. B 95060201

[14] Rozenbaum E B, Ganeshan S and Galitski V 2017 Phys. Rev. Lett. 118086801

[15] Chen X, Zhou T, Huse D A and Fradkin E 2017 Ann. Phys., Lpz. 5291600332

[16] Fan R, Zhang P, Shen H and Zhai H 2017 Sci. Bull. 62707

[17] Lewis-Swan R, Safavi-Naini A, Bollinger J J and Rey A M 2019 Nat. Commun. 101

[18] Gärttner M, Hauke P and Rey A M 2018 Phys. Rev. Lett. 120 040402

[19] Halpern N Y, Bartolotta A and Pollack J 2019 Commun. Phys. 21

[20] Larkin A and Ovchinnikov Y N 1969 Sov. Phys. JETP 281200

[21] Aleiner I L and Larkin A I 1996 Phys. Rev. B 5414423

[22] Li J, Fan R, Wang H, Ye B, Zeng B, Zhai H, Peng X and Du J 2017 Phys. Rev. X 7031011

[23] Wei K X, Ramanathan C and Cappellaro P 2018 Phys. Rev. Lett. 120070501

[24] Niknam M, Santos L F and Cory D G 2020 Phys. Rev. Res. 2 013200

[25] Gärttner M, Bohnet J G, Safavi-Naini A, Wall M L, Bollinger J J and Rey A M 2017 Nat. Phys. 13781

[26] Landsman K A, Figgatt C, Schuster T, Linke N M, Yoshida B, Yao N Y and Monroe C 2019 Nature 56761

[27] Kohmoto M, Sutherland B and Tang C 1987 Phys. Rev. B 35 1020

[28] Kohmoto M 1983 Phys. Rev. Lett. 511198

[29] Tang C and Kohmoto M 1986 Phys. Rev. B 342041

[30] Goldman A I and Kelton R F 1993 Rev. Mod. Phys. 65213

[31] Aubry S and André G 1980 Ann. Isr. Phys. Soc. 318

[32] Harper P G 1955 Proc. Phys. Soc. A 68874

[33] Roati G, D'Errico C, Fallani L, Fattori M, Fort C, Zaccanti M, Modugno G, Modugno M and Inguscio M 2008 Nature 453 895

[34] Lahini Y, Pugatch R, Pozzi F, Sorel M, Morandotti R, Davidson N and Silberberg Y 2009 Phys. Rev. Lett. 103013901

[35] Lye J, Fallani L, Modugno M, Wiersma D, Fort C and Inguscio M 2005 Phys. Rev. Lett. 95070401

[36] Lucioni E et al 2011 Phys. Rev. Lett. 106230403

[37] Oganesyan V and Huse D A 2007 Phys. Rev. B 75155111

[38] Pal A and Huse D A 2010 Phys. Rev. B 82174411

[39] Iyer S, Oganesyan V, Refael G and Huse D A 2013 Phys. Rev. B 87134202

[40] Schreiber M, Hodgman S S, Bordia P, Lüschen H P, Fischer M H, Vosk R, Altman E, Schneider U and Bloch I 2015 Science 349842

[41] Deng X, Ray S, Sinha S, Shlyapnikov G and Santos L 2019 Phys. Rev. Lett. 123025301

[42] Roy N and Sharma A 2021 Phys. Rev. B 103075124

[43] Lieb E H and Robinson D W 1972 Commun. Math. Phys. 28 251

[44] Sekino Y and Susskind L 2008 J. High Energy Phys. JHEP10(2008)065

[45] Sachdev S 2015 Phys. Rev. X 5041025 
[46] Huang Y, Zhang Y-L and Chen X 2017 Ann. Phys., Lpz. 529 1600318

[47] Chávez-Carlos J, López-del Carpio B, Bastarrachea-Magnani M A, Stránskỳ P, Lerma-Hernández S, Santos L F and Hirsch J G 2019 Phys. Rev. Lett. 122024101

[48] Jalabert R A, García-Mata I and Wisniacki D A 2018 Phys. Rev. E 98062218

[49] Lin C-J and Motrunich O I 2018 Phys. Rev. B 97144304

[50] Bao J and Zhang C-Y 2019 arXiv:1901.09327

[51] Fortes E M, García-Mata I, Jalabert R A and Wisniacki D A 2020 arXiv:2004.14440

[52] Yan H, Wang J-Z and Wang W-G 2019 Commun. Theor. Phys. 711359

[53] Riddell J and Sørensen E S 2019 Phys. Rev. B 99054205

[54] Riddell J and Sørensen E S 2020 Phys. Rev. B 101024202

[55] Lee J, Kim D and Kim D-H 2019 Phys. Rev. B 99184202

[56] Bordia P, Alet F and Hosur P 2018 Phys. Rev. A 97030103

[57] Modugno M 2009 New J. Phys. 11033023

[58] Bugeaud Y 2008 Math. Ann. 341677

[59] Cohn H 2006 Am. Math. Mon. 11357

[60] Roy N and Sharma A 2019 Phys. Rev. B 100195143
[61] McGinley M, Nunnenkamp A and Knolle J 2019 Phys. Rev. Lett. 122020603

[62] Peschel I 2003 J. Phys. A: Math. Gen. 36 L205

[63] Roy N and Sharma A 2018 Phys. Rev. B 97125116

[64] Roósz G, Divakaran U, Rieger H and Iglói F 2014 Phys. Rev. B 90184202

[65] Hetterich D, Serbyn M, Domínguez F, Pollmann F and Trauzettel B 2017 Phys. Rev. B 96104203

[66] Lerose A and Pappalardi S 2020 Phys. Rev. Res. 2012041

[67] Buyskikh A S, Fagotti M, Schachenmayer J, Essler F and Daley A J 2016 Phys. Rev. A 93053620

[68] Modak R and Nag T 2020 Phys. Rev. Res. 2012074

[69] Falcon S 2014 Appl. Math. 052226

[70] Lin C-J and Motrunich O I 2018 Phys. Rev. B 98134305

[71] Chen B, Hou X, Zhou F, Qian P, Shen H and Xu N 2020 arXiv:2001.06333

[72] Dağ C B, Sun K and Duan L-M 2019 Phys. Rev. Lett. 123 140602

[73] Lewis-Swan R J, Muleady S R and Rey A M 2020 Phys. Rev. Lett. 125240605

[74] Nie X et al 2020 Phys. Rev. Lett. 124250601 